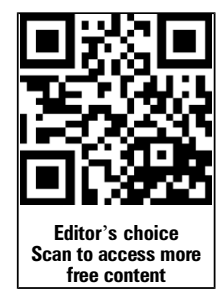

For numbered affiliations see end of article.

\section{Correspondence to} Dr TK Mattingly, London Health Sciences Center, Western University, University Hospital, 339 Windermere Road, London, ON, Canada N6A 5A5;

tmattingly206@gmail.com

Portions of this work have been presented in abstract form at the Canadian Neurological Sciences Foundation 49th Annual Meeting, Banff, Alberta, 2014, and the American Association of Neurological Surgeons Annual Meeting, New Orleans, Louisiana, 2013.

Received 15 November 2014 Revised 21 January 2015 Accepted 26 January 2015 Published Online First 12 February 2015

\title{
Catheter based selective hypothermia reduces stroke volume during focal cerebral ischemia in swine
}

\author{
Thomas K Mattingly, ${ }_{1}^{1}$ Lynn M Denning, ${ }^{1}$ Karen L Siroen, ${ }^{1}$ Barb Lehrbass, ${ }^{2}$ \\ Pablo Lopez-Ojeda, ${ }^{1,2}$ Larry Stitt, ${ }^{3}$ David M Pelz, ${ }^{1,2}$ Sumit Das, ${ }^{4}$ Lee-Cyn Ang, ${ }^{4}$ \\ Donald H Lee, ${ }^{1,2}$ Stephen P Lownie ${ }^{1,2}$
}

To cite: Mattingly TK, Denning LM, Siroen KL, et al. I Neurolntervent Surg 2016;8:418-422.

\section{ABSTRACT}

Background Total body hypothermia is an established neuroprotectant in global cerebral ischemia. The role of hypothermia in acute ischemic stroke remains uncertain. Selective application of hypothermia to a region of focal ischemia may provide similar protection with more rapid cooling and elimination of systemic side effects. We studied the effect of selective endovascular cooling in a focal stroke model in adult domestic swine.

Methods After craniotomy under general anesthesia, a proximal middle cerebral artery branch was occluded for $3 \mathrm{~h}$, followed by $3 \mathrm{~h}$ of reperfusion. In half of the animals, selective hypothermia was induced during reperfusion using a dual lumen balloon occlusion catheter placed in the ipsilateral common carotid artery. Following reperfusion, the animals were sacrificed. Brain MRI and histology were evaluated by experts who were blinded to the intervention.

Results 25 animals were available for analysis. Using selective hypothermia, hemicranial temperature was successfully cooled to a mean of $26.5^{\circ} \mathrm{C}$. Average time from start of perfusion to attainment of moderate hypothermia $\left(<30^{\circ} \mathrm{C}\right)$ was $25 \mathrm{~min}$. Mean MRI stroke volumes were significantly reduced by selective cooling ( $0.050 \pm 0.059$ control, $0.005 \pm 0.011$ hypothermia (ratio stroke:hemisphere volume) $(p=0.046)$. Stroke pathology volumes were reduced by $42 \%$ compared with controls ( $p=0.256$ ).

Conclusions Selective moderate hypothermia was rapidly induced using endovascular techniques in a clinically realistic swine stroke model. A significant reduction in stroke volume on MRI was observed. Endovascular selective hypothermia can provide neuroprotection within time frames relevant to acute ischemic stroke treatment.

\section{INTRODUCTION}

Hypothermia is the oldest and best studied neuroprotectant. Animal and human studies have established its benefit. ${ }^{1-13}$ Pioneering surgical investigators recognized the potential for deep whole body hypothermia to protect against focal ischemia, but noted significant side effects. ${ }^{14-16}$ These challenges led a few early investigators to pursue selective hypothermia applied only to the region of ischemia. ${ }^{17-19}$ Advances in endovascular technology have rekindled interest in the selective approach. Human trials, such as Cooling for Acute Ischemic Brain Damage (COOL AID) and Intravascular Cooling for the Treatment of Stroke-Longer window (ICTuS-L), as well as animal studies, suggest that applying selective hypothermia using an endovascular approach could provide rapid induction of therapeutic hypothermia within a meaningful time frame in focal cerebral ischemia. ${ }^{7} 9$ 18-22

In this study, we investigated catheter based selective brain hypothermia in an adult domestic swine model of stroke. We wished to determine whether moderate selective brain hypothermia could be rapidly induced using percutaneous endovascular technology, and whether stroke volumes could be reduced compared with a control group. Our model consisted of temporary transcranial occlusion of the middle cerebral artery (MCA) followed by percutaneous placement of a novel endovascular cooling catheter to provide selective hemicranial cooling during reperfusion.

\section{METHODS}

\section{Setup and craniotomy}

Under an approved animal research protocol (Schulich School of Medicine and Dentistry Animal Use Protocol 2009-079) and following Canadian Council of Animal Care guidelines, 28 YorkshireLandrace-cross pigs $(50 \mathrm{~kg})$ were assigned to normothermia or hypothermia in a 1:1 alternating fashion. Following acclimation in an approved vivarium for $48 \mathrm{~h}$, animals were anesthetized using 1-2 $\mathrm{mL}$ of intramuscular Telazol (Fort Dodge, Iowa, USA), intubated, and maintained with a nitrous oxide-isoflurane mixture. Arterial and central venous monitoring was obtained via bilateral transfemoral 6 F sheath placement, and a right fronto-orbital craniotomy was performed. ${ }^{23}$ After opening the arachnoid to release CSF, the head of the bed was elevated to provide maximal brain relaxation. Mannitol was avoided to eliminate any confounding neuroprotective effects. ${ }^{2425}$ Under high surgical magnification, a temporary aneurysm clip was applied to one of the two to three MCA branches running beneath the posterior frontal lobe. After $3 \mathrm{~h}$ of occlusion, the clip was removed and the scalp closed.

\section{Selective brain cooling}

Hypothermia animals were turned supine immediately following skin closure. Under fluoroscopy, an arterial $6 \mathrm{~F}$ sheath was exchanged over a series of dilators for the $14 \mathrm{~F}$ outer flow lumen (OFL) of the TwinFlo catheter (Thermopeutix Inc, San Diego, California, USA) (figure 1). The OFL was positioned in the descending thoracic aorta. Thereafter 


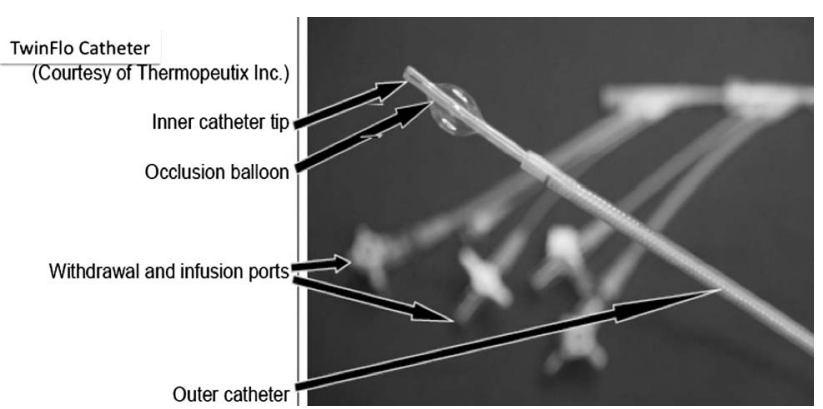

Figure 1 Thermopeutix TwinFlo catheter. Distal end of the inner and outer catheters showing the $14 \mathrm{~F}$ aortic outflow catheter with the $9.5 \mathrm{~F}$ carotid inflow balloon occlusion catheter.

activated clotting time was maintained at least two times normal using intravenous heparin. The $9.5 \mathrm{~F}$ inner flow lumen (IFL), a dual lumen balloon catheter, was introduced through the OFL and navigated into the right common carotid artery (ipsilateral to the ischemic hemisphere). The OFL and IFL ports were attached to an extracorporeal circuit (Maquet Rotoflow pump, Jostra chiller, Medtronic Myotherm heat exchanger), and extracorporeal circulation was established using a starting temperature of $25^{\circ} \mathrm{C}$. The IFL balloon was then inflated to radiographic occlusion of the artery. Pressure monitoring from the distal end of the IFL and inflow rates at the extracorporeal circuit were checked to avoid excessive perfusion pressures or flow rates. Inflow temperature was decreased in increments of $5^{\circ} \mathrm{C}$ to rapidly achieve hypothermia while maintaining hemodynamic stability. Extracorporeal cooling via the common (rather than the internal) carotid artery was deliberate in order to cool the brain using both direct and indirect pathways. Hemodynamic parameters (core temperature, heart rate, mean arterial blood pressure) and arterial blood gases (oxygen, hemoglobin, glucose, $\mathrm{pH}$ ) were continuously evaluated. The decision as to the depth of hypothermia was made on clinical grounds, based on the animal's hemodynamic stability. Perfusion was terminated once $3 \mathrm{~h}$ of reperfusion was completed.

\section{Temperature measurements}

Core temperatures were measured via rectal and esophageal thermometers. Nasal temperatures were used as a surrogate for brain temperature to avoid trauma from intraparenchymal probe insertion during the full anticoagulation required for the endovascular procedure. ${ }^{7}$ In the first selective hypothermia case, we found that subdural temperature measurements were within $2^{\circ} \mathrm{C}$ of transnasal measurements.

\section{Euthanasia, brain fixation, and analysis}

Following $3 \mathrm{~h}$ of reperfusion, the animal was euthanized with $108 \mathrm{mg} / \mathrm{kg}$ of Euthanyl Forte (Bimeda-MTC, Cambridge, Ontario, Canada). Fixation of the brain in situ with $500 \mathrm{~mL}$ of $10 \%$ neutral buffered formalin (EMD, Baltimore, Maryland, USA) was achieved using the IFL balloon catheter. The brain was removed for imaging and histology. MRI was performed postmortem and reviewed by a neuroradiologist blinded to the intervention. T2 and T1 three-dimensional sagittal images were obtained (T2=TR9700, TE 82.2 and TE 143 (142.7), $2 \mathrm{~mm}$ slice thickness, $16 \mathrm{~cm}$ FOV, $256 \times 256$ matrix; T1=TR 8.5 , TE $3.5,1.2 \mathrm{~mm}$ slice thickness, $12 \mathrm{~cm}$ FOV, $256 \times 256$ matrix). T2 data were used because diffusion information was lost at death. The area of T2 signal abnormality on each axial slice was determined using Osirix. Contiguous axial slices with T2 signal abnormality were summated to provide a total volume. An identical method of calculating hemispheric volume, excluding the midbrain, was performed, and the ratio of stroke volume to hemisphere volume was determined as a percentage. Next, the fixed cerebrum was sliced into $1 \mathrm{~cm}$ thick coronal slices and placed in cassettes for paraffin embedding. Pathological examination was conducted by a neuropathology team blinded to the intervention. Coronal slices of the cerebrum were stained with hematoxylin and eosin. ${ }^{7}$ Ischemic change was defined as the presence of both dark neurons and perineuronal vacuolation. The area of the ischemic change was determined manually, and then multiplied by the block thickness to give a volume.

\section{Statistics}

In the initial sample size calculation, it was estimated that $70 \%$ of animals in the normothermic group would have infarction of at least $20 \%$ of the cerebral hemisphere volume. Assuming that selective hypothermia provides a marked reduction in the proportion infarcted, from $70 \%$ to $<30 \%$ (or $6 / 20$ animals), according to Fisher's exact test, the two tailed $\mathrm{p}$ value will be $<0.02$ and the association between hypothermia and reduction in stroke will be considered statistically significant. Thus we initially planned for 20 animals in each group.

Hemodynamic parameters and arterial blood-gas values were compared using analysis of covariance. Changes in core temperature were compared using paired t tests. Between group comparisons of stroke volume were made using unpaired t tests or, where variances were unequal, using a Wilcoxon two sample test. Data were analyzed by an independent statistician using SAS V.9.3, and $\mathrm{p}$ values $<0.05$ were considered statistically significant.

\section{RESULTS}

Twenty-eight pigs were studied. One died due to pre-existing pulmonary artery stenosis and hypertrophic cardiomyopathy. Two pigs were unusable due to inadvertent brain contusions during exposure, and no ischemia was induced. Twenty-five animals were available for analysis, consisting of 13 control and 12 selective hypothermia cases. There was one aortic dissection in the selective hypothermia arm. Differences in systemic parameters between controls and hypothermic animals were seen only in core temperature $(\mathrm{p}<0.001)$ and in $\mathrm{pH}(\mathrm{p}<0.001)$ during the $3 \mathrm{~h}$ reperfusion-cooling phase of the experiment. However, core temperatures did not decrease below mild hypothermic levels $\left(32-34^{\circ} \mathrm{C}\right.$ ) (table 1$)$. The $\mathrm{pH}$ was $7.34 \pm 0.18$ in the hypothermia cohort compared with $7.44 \pm 0.03$ in the control group.

\section{Selective brain cooling}

The mean ipsilateral (right) hemicranial temperature decreased from $38^{\circ} \mathrm{C}$ to $26^{\circ} \mathrm{C}$, while the contralateral (left) temperature decreased to $31.6^{\circ} \mathrm{C}$ during selective hypothermic perfusion (table 1). The average time to place the catheters and establish extracorporeal circulation was over $1 \mathrm{~h}$ but varied widely

Table 1 Temperature during selective hypothermia

\begin{tabular}{|c|c|c|c|c|}
\hline & $3 \mathrm{~h}$ & $6 \mathrm{~h}$ & Difference & $\mathrm{p}$ Value \\
\hline Core & $38.02 \pm 0.64$ & $34.00 \pm 1.29$ & $4.02 \pm 1.13$ & $<0.001$ \\
\hline Right & $38.02 \pm 0.72$ & $26.53 \pm 2.55$ & $11.48 \pm 2.70$ & $<0.001$ \\
\hline Left & $37.76 \pm 1.03$ & $31.57 \pm 3.67$ & $6.09 \pm 3.65$ & $<0.001$ \\
\hline
\end{tabular}


Table 2 Selective hypothermia parameters

\begin{tabular}{lcl}
\hline & Median $($ Q1, Q3) & Min, Max \\
\hline Setup time $(\mathrm{min})^{*}$ & $52.0(34,75)$ & 22,144 \\
Temp nadir $\left({ }^{\circ} \mathrm{C}\right)$ & $23.70(23.3,25.3)$ & $21.8,27.5$ \\
Time to $<30^{\circ} \mathrm{C}(\mathrm{min}) \dagger$ & $15.0(13,35)$ & 6,65 \\
Total cooling $(\mathrm{min}) \ddagger$ & $125.0(87,134)$ & 36,150 \\
Total time to $<30^{\circ} \mathrm{C}(\mathrm{min}) \S$ & $87.0(52,109)$ & 47,155 \\
\hline
\end{tabular}

${ }^{*}$ Time from skin closure needed to exchange catheters, position the outer flow lumen and inner flow lumen appropriately, and connect and purge the extracorporeal circulation.

tTime required to achieve moderate hypothermia once extracorporeal perfusion was started.

$¥$ Sum of setup time and extracorporeal perfusion time.

$\S$ Sum of setup time and time to achieve moderate hypothermia once extracorporeal perfusion was started.

(table 2). Once extracorporeal circulation was established, moderate hypothermia $\left(<30^{\circ} \mathrm{C}\right)$ was routinely achieved within $30 \mathrm{~min}$. The total time from the start of cerebral reperfusion due to removal of the aneurysm clip to attainment of moderate hypothermia was $<1.5 \mathrm{~h}$.

\section{MRI and pathological stroke volumes}

The ratio of stroke volume to hemispheric volume of the hypothermia group $(0.005 \pm 0.011)$ was significantly smaller than that of the control group $(0.050 \pm 0.059)$ by MRI T2 imaging. The difference was statistically significant $(\mathrm{p}<0.05)$ (table 3 ).

Mean histologic stroke volumes were substantially reduced with hypothermia to $58 \%$ of controls. Due to wide variability, this difference did not attain significance $(p=0.256)$.

\section{DISCUSSION}

\section{Selective endovascular cooling for acute ischemic stroke}

Animal studies of hypothermia have shown benefit during both global and focal cerebral ischemia. ${ }^{1}{ }^{2}{ }^{8-10}$ Human trials have demonstrated that hypothermia is beneficial in ameliorating global brain ischemia following cardiac arrest. ${ }^{26} 27$ Whether there is any role for hypothermia after focal ischemic stroke is uncertain. $^{28}$ Whole body surface cooling requires hours to perform and is therefore impractical for acute stroke care. Both COOL AID and ICTUS-L trials achieved rapid hypothermia via endovascular transvenous cooling. ${ }^{20} 21$ Whole body hypothermia is limited to mild hypothermia $\left(>30^{\circ} \mathrm{C}\right)$ by cardiac arrhythmia. ${ }^{14}$ However, significant reductions in $\mathrm{CMRO}_{2}$ do not occur until moderate-deep hypothermia $\left(25-28^{\circ} \mathrm{C}\right) .{ }^{18}$ Selective transarterial hypothermia can overcome the limitations of whole body cooling and has been used as neuroprotection during brain surgery. ${ }^{6}$ It can achieve moderate-deep hypothermia while maintaining core temperatures at physiologically acceptable levels. ${ }^{7}$ 17-19 22 When combined with selective hypothermia, which offers a degree of cooling not achievable with whole body methods, endovascular technology could be a 'neuroprotective bridge' in recanalization. ${ }^{28-30}$

Table 3 Stroke volumes by pathology and MRI

\begin{tabular}{lccc}
\hline & Control & Neuroprotection & p Value \\
\hline Path volume $\left(\mathrm{cm}^{3}\right)$ & $0.99 \pm 1.00$ & $0.57 \pm 0.76$ & 0.256 \\
MRI volume $($ ratio stroke: & $0.050 \pm 0.059$ & $0.005 \pm 0.011$ & 0.046 \\
hemisphere) $(\times 100=\%)$ & & & \\
\hline Values are mean $\pm S D$. & & &
\end{tabular}

Our study demonstrated that a purely endovascular approach can achieve moderate hypothermia within $30 \mathrm{~min}$. Previous selective hypothermia studies utilized open surgical femoralcarotid bypass, and all reported faster rate and greater depth of hypothermia than in human trials of endovascular transvenous hypothermia. $^{6-9} \quad 11 \quad 13 \quad 17 \quad 19$ In COOL AID, mean time to achieve a target of $33^{\circ} \mathrm{C}$ was $77 \mathrm{~min}$, while in ICTUS-L, mean time was $138 \mathrm{~min}$ after catheter placement. In both studies, some patients were $8 \mathrm{~h}$ post stroke before mild hypothermia was achieved, while target temperatures were only in the mild hypothermia range. Our study demonstrated that a combination of endovascular technology with selective hypothermia allows significant hypothermia $\left(<30^{\circ} \mathrm{C}\right)$ within a meaningful time frame for acute stroke intervention.

\section{Effectiveness of selective hypothermia}

Selective hypothermia reduced stroke volumes despite a significant delay in intervention ( $3 \mathrm{~h}$ of ischemia, mean $4.5 \mathrm{~h}$ from ischemia onset until moderate hypothermia). This was statistically significant for MRI stroke volumes. A similar trend was found for pathological stroke volumes but was not significant due to the wide variation in stroke volumes in our model. This may be a feature of our model, but is also seen in human clinical studies. ${ }^{20}$ Previous investigations of selective hypothermia examined subjects concurrently exposed to ischemia and hypothermia and have shown neuroprotective effects by clinical examination, radiology, and histology. ${ }^{8-9} 17$ Studies in rodents suggest that stroke may be ameliorated even when hypothermia is applied in a delayed fashion. ${ }^{1-2} 12$ Proposed mechanisms for this benefit include the protection of 'Penumbra' neurons at the peripheral zone of ischemia, suppression of excitatory neurotransmitters, and suppression of leukotrienes. ${ }^{10}$ During reperfusion with hypothermia, there are measurable reductions in ischemic metabolites, such as lactate and high energy phosphates, and reduction in one form of blood-brain barrier disruption. ${ }^{2} 4$

The efficacy of cooling during reperfusion after stroke in higher order mammals is less well studied. In clinical trials and practical experience in humans, strokes are seen and intervened on after many hours of ischemia. For selective hypothermia to be relevant for acute ischemic stroke, benefit must be shown after prolonged ischemia. Our ischemic insult of $3 \mathrm{~h}$ is the longest in a large animal study of selective hypothermia. Mean time from ischemic insult to target moderate hypothermia $\left(<30^{\circ} \mathrm{C}\right)$ was almost $4.5 \mathrm{~h}$, including a mean setup time of $1 \mathrm{~h}$ and time to target temperature of $25 \mathrm{~min}$. Despite these delays, significant benefit by imaging criteria was seen for selective hypothermia during reperfusion.

\section{Tolerance of selective hypothermia}

A concern in therapeutic hypothermia is the avoidance of complications, including arrhythmias, coagulopathy, and pneumonia. Most human therapeutic hypothermia protocols are limited to $32-34^{\circ} \mathrm{C}$. The degree of systemic cooling seen in our study (mean $34^{\circ} \mathrm{C}$ ) agrees with prior work, and should be tolerable, but measures of this, such as arrhythmia frequency, were not evaluated. $^{722}$ Coagulopathy was not assessed as heparin anticoagulation was used during the procedure. A mild but significant decrease was seen in arterial $\mathrm{pH}$. The stability of other factors, such as mean arterial blood pressure, blood oxygenation, and hemoglobin, indicates that placing the endovascular device and utilizing extracorporeal perfusion to induce selective hypothermia did not destabilize the subjects. 


\section{Study limitations}

The choice of stroke model resulted in three key limitations. First, under lengthy periods of anesthesia, domestic swine develop myocardial depression resulting in frequent use of inotropic support. Interruption of cooling was sometimes required in order to stabilize hemodynamics. Second, the decision to occlude one of several MCA branches created small strokes which, in combination with collateral pathway variation, may have contributed to the wide variance in stroke volumes. Third, reperfusion of the MCA territory was only confirmed by visual inspection. Cerebral perfusion was not measured, and vessel patency following reperfusion was not ensured by indocyanine green or conventional angiography.

The timing of MRI introduces uncertainty. As MRI was postmortem in this study, T1 and T2 weighted sequences were used. ${ }^{31}$ Diffusion weighted imaging is the gold standard in stroke, but requires a live animal. The experimental results may reflect changes in reperfusion damage, rather than stroke damage. We measured stroke volumes accurately, but microscopic effects (changes in local chemical substrates) and macroscopic effects (edema or raised intracranial pressure) due to reperfusion were not evaluated. Thus potential effects of hypothermia on reperfusion injury might have been missed.

\section{Future directions}

This study raises intriguing questions regarding the efficacy of hypothermia in the face of acute stroke. It would be useful to assess the effect of clipping all MCA branches in terms of stroke volume and variability. The timing of reperfusion deserves clarification. Selective hypothermia could be effective in reducing stroke volumes, even with no recanalization. Finally, performing MRI prior to sacrifice would allow diffusion weighted imaging measurements of stroke volume. Logistical hurdles to this are substantial, as the entire operation, including life support and catheter, would need to be MRI compatible, and transport of an animal potentially on pressors might result in a premature end to the experiment.

\section{CONCLUSION}

This is the first study to investigate endovascular technology specifically designed to produce selective brain hypothermia. We have demonstrated that moderate selective hypothermia is rapidly achievable through a completely percutaneous endovascular approach. Times and temperatures correspond well with the results of other animal studies of selective hypothermia using open femoral to carotid bypass. We found a significant reduction in stroke volume by imaging with our intervention. These results suggest that modern endovascular technology, already in place for stroke recanalization, may be a vehicle for hypothermic neuroprotection which in turn may improve stroke outcomes following recanalization.

\section{Author affiliations}

${ }^{1}$ Department of Clinical Neurological Sciences, Western University, London, Ontario, Canada

${ }^{2}$ Department of Medical Imaging, Western University, London, Ontario, Canada

${ }^{3}$ Department of Statistics, Stitt Statistical Services, Inc, London, Ontario, Canada

${ }^{4}$ Department of Pathology, Western University, London, Ontario, Canada

Acknowledgements The authors thank Ron Solar PhD and Glen Lieber of Thermopeutix Inc for technical support and data.

Contributors SPL, LMD, KLS, DMP, and TKM: conception and design. All authors: acquisition, analysis, and interpretation of the data, revising the manuscript, final approval of the published manuscript, and accountability. TKM, LMD, SPL, and LS: drafting the manuscript.
Funding This work was supported by the Heart and Stroke Foundation of Canada (GIA NA 7273).

Competing interests Thermopeutix Inc (San Diego, California, USA) provided material support consisting of Twin-Flo catheters and some data collection on catheter performance.

Ethics approval The study was conducted under an approved animal research protocol (Schulich School of Medicine and Dentistry Animal Use Protocol 2009-079) and following Canadian Council of Animal Care guidelines.

Provenance and peer review Not commissioned; externally peer reviewed.

Data sharing statement This study is ongoing. All raw and analyzed data related to this published cohort will be made available upon request to the corresponding or senior authors.

\section{REFERENCES}

1 Goto Y, Kassell NF, Hiramatsu K, et al. Effects of intraischemic hypothermia on cerebral damage in a model of reversible focal ischemia. Neurosurgery 1993;32:980-4

2 Huang ZG, Xue D, Preston E, et al. Biphasic opening of the blood-brain barrier following transient focal ischemia: effects of hypothermia. Can J Neurol Sci 1999;26:298-304.

$3 \mathrm{Ji} \mathrm{Y,} \mathrm{Hu} \mathrm{Y,} \mathrm{Wu} \mathrm{Y,} \mathrm{et} \mathrm{al.} \mathrm{Therapeutic} \mathrm{time} \mathrm{window} \mathrm{of} \mathrm{hypothermia} \mathrm{is} \mathrm{broader} \mathrm{than}$ cerebral artery flushing in carotid saline infusion after transient focal ischemic stroke in rats. Neurol Res 2012:34:657-63.

4 Kozlowski P, Buchan AM, Tuor UI, et al. Effect of temperature in focal ischemia of rat brain studied by 31P and $1 \mathrm{H}$ spectroscopic imaging. Magn Res Med 1997;37:346-54

5 Lawton MT, Raudzens PA, Zabramski JM, et al. Hypothermic circulatory arrest in neurovascular surgery: evolving indications and predictors of patient outcome. Neurosurgery 1998;43:10-20.

6 Lownie SP, Menkis AH, Craen RA, et al. Extracorporeal femoral to carotid artery perfusion in selective brain cooling for a giant aneurysm. J Neurosurg 2004;100:343-7.

7 Mori K, Saito J, Kurata Y, et al. Rapid development of brain hypothermia using femoral-carotid bypass. Acad Emerg Med 2001;8:303-8.

8 Mori K, Itoh Y, Saito J, et al. Post-resuscitative hypothermic bypass reduces ischemic brain injury in swine. Acad Emerg Med 2001;8:937-45.

9 Ohta T, Kuroiwa T, Sakguchi I, et al. Selective hypothermic perfusion of the canine brain. Neurosurgery 1996;38:1211-15.

10 Onesti ST, Baker CJ, Sun PA, et al. Transient hypothermia reduces focal ischemic brain injury in the rat. Neurosurgery 1991;29:369-71.

11 Schwartz AE, Finck D, Stone JG, et al. Delayed selective cerebral hypothermia decreases infarct volume after reperfused stoke in baboons. J Neurosurg Anesth 2011:23:124-30.

12 Xue D, Huang Z-G, Smith KE, et al. Immediate or delayed mild hypothermia prevents focal cerebral infarction. Brain Res 1992;587:66-72.

13 Zhang I, Cheng $H$, Shi J, et al. Focal epidural cooling reduces the infarction volume of permanent middle cerebral artery occlusion in swine. Surg Neurol 2007;67:117-21

14 Bigelow WG, Lindsay WK, Greenwood WF. Hypothermia: Its possible role in cardiac surgery: an investigation of factors governing survival in dogs at low body temperatures. Ann Surg 1950;132:849-66.

15 Botterell EH, Lougheed WM, Scott JW, et al. Hypothermia, and interruption of carotid, or carotid and vertebral circulation in the surgical management of intracranial aneurysms. J Neurosurg 1956;13:1-42.

16 Drake CG, Barr HWK, Coles JC, et al. The use of extracorporeal circulation and profound hypothermia in the treatment of ruptured intracranial aneurysm. J Neurosurg 1964;21:575-81.

17 Verdura J, White RJ, Albin MS. Profound selective hypothermia and arrest of arterial circulation to the dog brain. JNS 1966;24:1002-6.

18 Lougheed WM, Kahn DS. Circumvention of anoxia during arrest of cerebral circulation for intracranial surgery. J Neurosurg 1955;12:226-39.

19 Williams BN, Turner EA. Report of 10 operations under local cerebral hypothermia. J Neurol Neurosurg Psychiatry 1970;33:647-55.

20 De Georgia MA, Krieger DW, Ahou-Chebl A, et al. Cooling for Acute Ischemic Brain Damage (COOL AID). Neurology 2004;63:312-17.

21 Hemmen TM, Raman R, Guluma K, et al. Intravenous thrombolysis plus hypothermia for acute treatment of ischemic stroke: final results. Stroke 2010;41:2265-70.

22 Schwartz $A E$, Stone JG, Finck $A D$, et al. Isolated cerebral hypothermia by single carotid artery perfusion of extracorporeally cooled blood in baboons. Neurosurgery 1996;39:577-82.

23 Imai $\mathrm{H}$, Konno K, Nakamura $\mathrm{M}$, et al. A new model of focal cerebral ischemia in the miniature pig. J Neurosurg (2 Suppl Ped) 2006;104:123-32. 
24 Korenkov Al, Pahnke J, Frei K, et al. Treatment with nimodipine or mannitol reduces programmed cell death and infarct size following focal cerebral ischemia. Neurosurg Rev 2000;23:145-50.

25 Luvisotto TL, Auer RN, Sutherland GR. The effect of mannitol on experimental cerebral ischemia, revisited. Neurosurgery 1996;38:131-8.

26 Holzer M, Bernard SA, Hachimi-Idrissi S, et al. Hypothermia for neuroprotection after cardiac arrest. Crit Care Med 2005;33:414-18.

27 Shankaran S, Laptook AR, Ehrenkranz RA, et al. Whole body hypothermia for neonates with hypoxic ischemic encephalopathy. New Engl J Med 2005:353:1574-84.
28 Yenari MA, Hemmen TM. Therapeutic hypothermia for brain ischemia. Where have we come and where do we go? Stroke 2010;41(Suppl 1):S72-74.

29 Froehler MT, Ovbiagele B. Therapeutic hypothermia for acute ischemic stroke. Expert Rev Cardiovasc Ther 2010:8:593-603.

30 Choi JH, Marshall RS, Neimark MA, et al. Selective brain cooling with endovascular intracarotid infusion of cold saline: a pilot feasibility study. AJNR 2010;31:928-34.

31 Knight RA, Dereski MO, Helpern JA, et al. Magnetic resonance imaging assessment of evolving focal cerebral ischemia: comparison with histopathology in rats. Stroke 1994;25:1252-61.

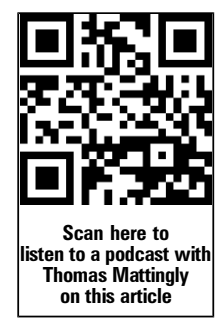

